\title{
Composite Materials: Characterization, Fabrication and Application-Research Challenges and Directions
}

\author{
Ahmad Varvani-Farahani \\ Received: 15 October 2009 / Accepted: 20 October 2009/Published online: 6 November 2009 \\ (C) Springer Science + Business Media B.V. 2009
}

Composite Materials have come to existence as long as the history of human being is known. Woods are natural composites surrounding mankind from the very first day. Animal bone as primitive natural composite was used for fishing, hunting and agricultural activities by human. Traditional bone knife of unknown age of $10000 \mathrm{BC}$ or earlier has been kept in The National History Museum. Mummification may be one of the very first humancomposite made which is documented in the history of ancient Egypt. Mummies were wrapped with strips of white linen with a thin layer of oil that protected the body from being damaged. The oldest known mummy found is dated $3000 \mathrm{BC}$. The world's largest composite structure manifesting the civilization in human history belonged to the city of Arg-e-Bam with area of approximately 180,000 square meters. The city of Arg-e-bam was made of bricks consist of straw and mud with a history as old as 2000 BC.

The last few decades of the second millennium faced human with more of challenges to develop advanced engineering materials. Organic-Matrix Composites (OMC) originated during World War II (1939-1945) in an effort by Aerospace Industry to produce materials with better resistance against corrosion and fatigue damage. When advanced composites technology was promoted in the early 1960s, the emphasis was an increased performance by means of reducing structural weight; very little attention was given to low-cost manufacturing. The demand for high performance composites was further aggrandized by the high cost of fuel which resulted from the energy crisis during the 1970s. Over 35 years, OMC have won an increasing mass fraction of aircrafts and spacecraft structures. The most widely used fibers in OMCs are fiber tows consist of thousands fine filaments arranged in a single bundle. Glass-Fiber-Reinforced Polymer Composites (GRPs) have found extensive applications including fiber-glass boats, pressure vessels, and airplane panels. Glass fibers are the earliest known fibers in advanced composites compatible with Polyester and Epoxy matrices. Boron-FiberReinforced Composites were used as structural components. Boron fibers were first developed and marketed in 1960s in the form of monofilaments. Boron-epoxy hybrids

\footnotetext{
A. Varvani-Farahani $(\square)$ Department of Mechanical \& Industrial Engineering, Ryerson University, 350 Victoria Street, Toronto, Ontario M5B 2K3, Canada

e-mail: avarvani@ryerson.ca
} 
are being used in golf clubs and tennis rackets. Carbon-Fiber-Reinforced Polymer Composites (CFRPs) are known for their strength, durability and light-weight. Application of CFRP includes sport goods and components in aerospace industry. Aramid-Fiber-Reinforced Composites are filament-wound for motor cases, gas pressure vessels, fixed wing commercial aircrafts and helicopters. Aramid fiber is a thermoplastic polymer when heated it decomposes. Aramid composites display far lower strength in compression than carbon composites. Metal-Matrix Composites (MMCs) were first originated in 1950 s and early 1960 s. The principal motivation was to dramatically extend the structural efficiency of metallic materials while retaining their high chemical inertness, high shear strength and high resistance at elevated temperatures. Development of high strength monofilaments (first Boron and then silicon carbide (SiC)) enabled significant efforts on fiber-reinforced MMCs throughout the 1960s. In early 1970s, recession resulted research funding cuts in MMC. In 1980s, major efforts included particle-reinforced, whisker-reinforced MMC of aluminum-based for automotive and aircraft components where a high temperature resistance was required. In 1990s, two major developments included discontinuously reinforced aluminum (DRA) and Titanium (DRTi) for elevated temperature use (ASM Composites Handbook, Vol.21, 2001). DRAs are great material choices for brake rotors for high-speed trains and rotating components in gas turbine engine.

In 2004, over 4 billion pounds consumption of composite materials by various industries in the USA shows a drastic increase of composites materials over other traditionally used materials of steel and aluminum alloys (Composites, Mac Neil-2005).Constructions and transportation infrastructure with over $50 \%$ is a leading industry in consumption of composite materials, while aircraft industry with consumption of only $1 \%$ is the lowest consumer of composite materials. While USA is a leading consumer of composite materials, Europe, and Japan respectively with over 3 and 2 billion pounds are the second and the third most widely users of composites (Report No. KRN-A41). A worldwide increasing trend of composite materials consumption verifies the superiority of these materials over other traditional materials which can plot future directions of engineering design and research activities on advanced composite materials.

Current active research areas in composite materials provide some insight into technologies that will mature to practical application in both the short and long terms. While modern composites are in their early stage of progress, there is several worldwide ongoing research themes which are developed based on today's emerging technological challenges in characterization, fabrication and application plotting the future directions for research activities in composite materials. Some of the crucial research themes include; nano-composites/nano-phase reinforcements, multifunctional composites/ structures with self-assessment and self-repair capacity, shortening design cycle for the exploration of various design concepts at reduced costs, bio-composites for implants, technology innovations leading to new and improved composite materials, composite materials resources and restrictions, sustainability of composite resources, recyclability and environmental issues, development of nondestructive inspection (NDI) that can reliably measure durability of composites, developing composites with higher resistance at elevated temperature, corrosion, wear and impact, generation of pertinent data bank and information on various aspects of composites.

The first International Conference on Composites: Characterization, Fabrication and Application (CCFA-1) held in Kish Island on December 2008 was organized by the present guest editor to bring together both engineers and researchers in the field to discuss the latest research challenges in composites as well as future research themes and directions. The plan 
will be to secure this conference during December of every two years held on beautiful tropical islands. The CCFA-1 addressed advanced emerging research topics in the field through 183 submitted articles.

The special issue contains pear-reviewed selected papers presented at CCFA-1 conference. The following section briefly highlights papers on characterization, fabrication and application of composite materials appeared in the current special issue in the journal of Applied Composite Materials. Of the 15 articles published in this issue, three are on the buckling analysis and delamination growth of composite laminates, one is on the modes of failure in sandwich beams, one is on the stress corrosion of basalt/epoxy composites, one is on the fatigue damage of FRP $(0 / \theta)$ composite laminates, one is on the friction, fade and wear characteristics of a polymer matrix composite, two are on the design, fabrication and FE analysis of composite parts, one is on the effect of the moisture absorption of recycled reinforced polypropylene composites, two are on the applications of GFRP composites for crack remediation in concrete structures under loading, and three are on the vibrational/ dynamic response of composite shells and sandwich panels.

On the buckling analysis of the composite plates, Ovesy et al. implemented the semianalytical finite strip method (FSM) developed based on Reddy's higher order plate theory (HOPT). HOPT method was compared with the semi-analytical finite strip methods; classical laminated plate theory (CLPT) and first-order shear deformation theory (FSDT) and was found to be more applicable for different plate thicknesses. They concluded that the HOPT semi-analytical FSM is a reliable method for the preliminary design of thick composite plates under buckling. Mohammadi et al. however employed Levy boundary conditions to analyze buckling of thin functionally graded (FG) rectangular plates. The analytical method included decoupling the stability equations of thin FG rectangular plates. The critical buckling loads for a FG plate with different boundary conditions were presented and the effects of aspect ratios, thickness to side ratios on the critical buckling loads were discussed. Hosseini-Toudeshky et al. developed an FE program in conjunction with the geometric non-linearity of the von karman to define the strain/displacement relations under buckling. They concluded that the buckling load/ mode, and the delamination growth are influenced by the size of delamination and the stacking sequence of the composite laminates.

Modes of damage on sandwich beams with unequal faces were analytically predicted by Sadighi and Saadati using the sandwich panel higher order theory (SPHOT) estimating both core compression and face stresses. They compared the results obtained by SPHOT, sandwich panel classical theory (SPCT) and available experimental values and showed that the higher order theory closely predicted failure loads and the corresponding damage modes.

Shokrieh and Memar reported that the stress corrosion influenced the mode of the final failure on basalt/epoxy composites. The strength and fracture energy of basalt/epoxy composites under stress corrosion and in the presence of 5\% Sulfuric acid degraded over time intervals. They reported that the magnitude of degradation on materials strength exponentially increased as the loading magnitude exceeded $50 \%$ of the ultimate strength. Features on the fracture surface of failed samples observed during their microscopic examination verified that stress corrosion influenced the mode of the final failure on composite samples.

The fatigue damage of composite plies was integrated as microcracks and damage progressed in matrix (Region I), matrix-fiber interface (Region II) and fiber (Region III) resulting in stiffness reduction of composite laminates over life cycles. Shirazi and VarvaniFarahani assessed fatigue damage of $\operatorname{FRP}(0 / \theta)$ composite laminates by integrating the fatigue damage values of $0^{\circ}$ and $\theta$ plies. A weighting factor $\eta$ has been introduced to partition the 
efficiency of load carrying plies of $0^{\circ}$ and $\theta$ in the $(0 / \theta)$ composite lamina. The analysis is believed to be extendable for other composite laminates at different lay-up/stacking conditions.

Bagheri et al. examined friction, fade and wear characteristics of a polymer matrix composite using a small-scale friction testing machine. PMC material consisted of phenolic resin, short carbon fiber, graphite, quartz, barite and steel fiber. Experimental results verified that carbon fibers played a significant role in the formation of friction film, which was closely related to the friction performance. Generated stable friction film on the brake pad surface resulted in an excellent friction stability with less wear.

Ghiasi et al. designed a carbon fiber bicycle stem interconnecting the structural and the manufacturing parameters in design with composite materials. The stem part included a complex geometry, small size and a hollow with a high quality surface finish manufactured using bladder assisted Resin Transfer Moulding (RTM). The comparison of their numerical results with their experimental design obtained after months of experiment confirmed the ability of multi-objective optimization method used by them in shortening the design process. Sabour and Foghani presented a new method to design a semi-composite pressure vessel known as hoop-wrapped composite cylinder using fuzzy decision making and finite element method. von Mises failure criterion in steel liner and Hoffman's failure criterion in the reinforced composite have been employed. The fuzzy decision maker calculated the thickness of steel liner and the number of composite layers. Ansys nonlinear FE was used to analyze the residual stress in steel liner. Results showed that carbon fibers were the most suitable choice to improve the strength of the composite pressure vessel while the lower weight of the vessel was expected.

Shakeri and Ghasemian investigated the moisture absorption of recycled newspaper fiber and recycled newspaper-glass fiber hybrid reinforced polypropylene composites for outdoor applications. Long-term water absorption and thickness swelling kinetics of the composites were investigated with water immersion. It was found that the water absorption and thickness swelling increased with recycled newspaper content and water immersion time before an equilibrium condition was achieved. Composites made from the recycled newspaper showed comparable results with those made of the hybrid fiber.

Sadjadi et al. investigated the application of GFRP composites for crack remediation in liquid containing concrete structures under monotonic loading. Cracked reinforced concrete specimens subjected to tension and cyclic flexural loading were repaired externally using GFRP sheets. The specimens were then subjected to hydrostatic pressure using a water pressure chamber at the crack location. Their examination on the liquid containing structures (LCS) was further discussed when the LCS was subjected to applied external loads such as earthquake. In a study by Kabir and Eshaghian the flexural performance of composite steel-concrete beam girders retrofitted with CFRP was studied. The three dimensional interactive Tsai-Wu failure criterion was implemented to retrofitted composite girder in order to identify the failure mode. In their studies, a detailed parametric study was carried out to investigate the effects of geometry and material characteristics on flexural performance of a composite section.

Vibrational behavior of cylindrical composite shells was analyzed by Hemmatnezhad et al. Their formulation was established based on Sanders' thin shell theory. The modal forms were assumed to have the axial dependency in the form of Fourier series whose derivatives were legitimized using Stoke's transformation. The influence of some commonly used boundary conditions, the effect of variations in shell geometrical parameters, changes in the stacking sequence of the constituent materials used on the shell frequencies and its corresponding mode shapes were discussed. Malekzadeh et al. utilized the first order shear deformation theory (FSDT) and Love's first approximation theory in the shell's equilibrium 
equations. The multilayer cylindrical shells were composed of hybrid composite materials and were subjected to lateral impulse loads. Equilibrium equations for free and forced vibration problems of the shells were solved using Galerkin method. They attributed the effect of material properties, fiber orientation and stacking sequence on the time response of the composite shell. A dynamic finite element (DFE) model was developed by Hashemi and Adique to analyze the vibrational response of three-layered sandwich beams. The governing differential equations of motion of the sandwich beam consisted of layers with dissimilar properties. Displacement fields were imposed such that the face layers follow the Rayleigh beam assumptions, while the core was governed by Timoshenko beam theory. The Quasi-Exact DFE formulation also outperformed the conventional FEM, which makes it useful in benchmarking other studies or the examination of high frequency response where FEM requires the use of large number of elements for a higher accuracy.

Acknowledgements As the guest editor, I would like to express my sincere appreciation and thanks to the authors and reviewers for their scientific contribution to this special issue. I wish to convey my gratitude to Editor-in-Chief, Dr. Peter Beaumont and Springer Publishing for their support and encouragement of this special issue. A special word of thanks goes to Ms. Lenny Gumapac for her kind attention and timely response to authors and reviewers. 